\title{
Editorial
}

\section{The Impact of Gender on Diagnosis and Treatment in Endocrine Surgery}

\author{
Peter E. Goretzki \\ Department of General, Visceral and Transplant Surgery, Charité - University Medicine Berlin, Berlin, Germany
}

When discussing gender and endocrine diseases, most readers may primarily think of sex hormone-specific diseases caused by dysregulation of endocrine active organs, where sex hormones are produced. In the actual volume of Visceral Medicine, however, we focused on endocrine surgery in general and asked whether gender plays an important role in the diagnosis and treatment of patients with thyroid, parathyroid, adrenal, and neuroendocrine tumors.

Distribution of tumors is different in males and females with a predominance of benign thyroid and parathyroid tumors in women. This is not the case in differentiated thyroid malignancies, where the prevalence is equal in both genders. However, even in tumors associated with the inherited MEN-1 syndrome, gender-specific differences occur. Thymic neuroendocrine tumors we almost exclusively detect in males only, whereas all other tumors are distributed equally in both sexes.

Besides differences in the prevalence of endocrine tumors in males and females, gender also affects the prog- nosis of patients with endocrine tumors. Research demonstrates a more favorable outcome and long-term survival in women with differentiated thyroid carcinoma when compared to men. In contrast to this, survival in patients with anaplastic thyroid cancer, with parathyroid cancer and most neuroendocrine tumors is similar in both sexes.

Investigating the long-term follow-up of patients with operated benign endocrine diseases, specific differences can be detected between sexes. Thus, more cardiac complications but less cerebrovascular complications occur in operated men with secondary hyperparathyroidism when compared to women.

All data presented here once again confirm that generalization in diagnosis and treatment of endocrine diseases, as necessarily applied to guidelines, should not disguise the need for an individual decision in each patient, which also has to consider the influence of gender on symptoms, possible perioperative complications, and long-term follow-up. 